\title{
El tiempo en la tragedia de Séneca
}

\section{Robert Sklenár \\ rsklenar@utk.edu \\ University of Tennessee, Knoxville, Estados Unidos}

Recepción: 05 Julio 2021

Aprobación: 06 Agosto 2021

Publicación: 01 Septiembre 2021

Cita sugerida: Sklenáŕ, R. (2021). El tiempo en la tragedia de Séneca. Auster, (26), e065. https://doi.org/10.24215/23468890e065

\begin{abstract}
Resumen: A través de un análisis de pasajes de Edipo, Medea y Tiestes de Séneca, este artículo intenta demostrar que las tragedias de Séneca mantienen la ética estoica del naturam sequi a la vez que rechazan el cosmos estoico racional y, con él, la base racionalista de la ética estoica. Los personajes trágicos senequeanos quedan así atrapados entre dos imperativos éticos - seguir la naturaleza y comportarse racionalmente- que son contradictorios en el universo que se ven forzados a habitar.
\end{abstract}

Palabras clave: Tiempo, Estoicismo, Cosmología, Irracionalidad, Ética.

\begin{abstract}
Through an analysis of passages from Seneca's Oedipus, Medea, and Thyestes, this paper endeavors to demonstrate that Seneca's tragedies retain the Stoic ethic of naturam sequi while rejecting the rational Stoic cosmos, and with it the rationalistic basis for Stoic ethics. Senecan tragic characters are thus trapped between two ethical imperatives-following nature and behaving rationally-which are contradictory in the universe that they are forced to inhabit.
\end{abstract}

Keywords: Time, Stoicism, Cosmology, Irrationality, Ethics.

En su obra maestra Time in Greek Tragedy, título al cual este ensayo rinde un humilde y consciente homenaje, Jacqueline de Romilly vincula la representación del tiempo en la tragedia griega al desarrollo de la conciencia de éste en la cultura griega durante el siglo V a. C. ${ }^{1}$ En esta obra, el tiempo es, para Esquilo, el instrumento de una teodicea que da cuenta no sólo del carácter inevitable de la justicia divina, sino también de las demoras en su ejecución; ${ }^{2}$ los héroes sofocleos, en cambio, luchan contra las incertidumbres y adversidades a las que el tiempo, en forma de vida humana, los somete, pues el tiempo ya no es "el medio de la justicia, sino la causa de la inestabilidad y la fragilidad de la vida humana"; ${ }^{3}$ finalmente, para Eurípides, "el tiempo ha perdido su dimensión teleológica para adquirir una realidad y una complejidad psicológicas." ${ }^{4}$ La representación del tiempo en la tragedia de Séneca, por su parte, está relacionada con la concepción estoica del tiempo, pero de una manera que se diferencia marcadamente de la madurez de conciencia acerca de éste que refleja la tragedia griega: al contrario, la representación del tiempo en la tragedia de Séneca es un rasgo distintivo de su relación antagónica con la herencia filosófica estoica.

En beneficio de la claridad, comenzaré con dos postulados. El primero es que Séneca tragicus y Séneca philosophus son el mismo autor, aunque se han propuesto y siguen proponiéndose argumentos convincentes contra esta suposición. ${ }^{5}$ Ya sea que lidiemos con uno o dos autores, es claro que el autor de las tragedias de Séneca no ignora las categorías estoicas de Séneca philosophus, sino que, en su lugar, las rechaza consciente y sistemáticamente; de este modo, la teoría de un solo autor se ve fundamentada por el hecho de que las 
tragedias están en constante diálogo con las obras filosóficas. Mi segundo postulado es limitar la discusión a aquellas obras de indiscutible autoría senequeana conforme a la primera suposición, y que asimismo sean indiscutiblemente tragedias. Por tanto omitiré de mi lectura el Hercules Oetaeus, que con bastante certeza no es de Séneca (en efecto, nada menos que una autoridad como John Fitch cuestiona abiertamente que el latín sea la primera lengua del autor de esta obra ${ }^{6}$ ); también omito la Octavia, sobre la cual se discute no sólo la autoría sino también su condición de tragedia, y la Apokolocyntosis, sobre la base de que, sea lo que fuere esta curiosa obra, decididamente no es una tragedia. Por último, a los fines de esta discusión y para evitar ciertas redundancias, sigo la cronología relativa de la tragedia de Séneca propuesta por Fitch, ${ }^{7}$ que delimita tres períodos de composición: temprano (Oedipus, Agamemnon, Phaedra), medio (Medea, Troades, Hercules Furens) y tardío (Thyestes, Phonissae). Un ejemplo representará cada uno de estos períodos: Oedipus, Medea y Thyestes.

Es realmente difícil, hay que admitirlo, ensamblar una teoría estoica del tiempo coherente, principalmente debido al estado fragmentario de las fuentes tempranas. En la obra filosófica de Séneca, nos enfrentamos al problema opuesto: un río de prosa que al mismo tiempo desborda y serpentea. Al contrario del método de la mayoría de los filósofos, Séneca philosophus se resiste casi a cada paso a una exposición sistemática, optando en cambio por un estilo que se asemeja a un fluir de conciencia avant la lettre. Para resumir no obstante, en la medida en que un resumen sea posible: los estoicos antiguos consideraban que el tiempo era incorpóreo e “infinitamente divisible hacia ambos lados, pasado y futuro, del 'restrictivo' presente." ${ }^{8}$ En sentido estricto, el 'presente' no existe; el tiempo es un continuum, coincidente con el universo cíclico que culmina en la ekpyrōsis, la conflagración que regenera el universo en su forma primigenia. Lo que experimentamos como presente es puramente una cuestión de percepción, ya que no existe un punto fijo que separe el futuro del pasado: uno es simplemente la continuación del otro (Crisipo, según lo que informa Estobeo, SVF II, 509). ${ }^{9}$ Para Séneca philosophus el tiempo se vuelve una cuestión moral. El texto central es, por supuesto, De brevitate vitae: non exiguum temporis habemus, sed multum perdimus ("No tenemos escasez de tiempo, sino que perdemos mucho," Brev., I, 3); non accipimus brevem vitam, sed facimus, nec inopes eius sed prodigi sumus ("No recibimos una vida breve, sino que la hacemos breve; ni estamos faltos de ella, sino que la derrochamos", Brev., I, 4). A primera vista, parece que Séneca admite el concepto de presente:

in tria tempora vita dividitur: quod fuit, quod est, quod futurum est. Ex iis quod agimus breve est, quod acturi sumus dubium, quod egimus certum. hoc est enim, in quod fortuna ius perdidit, quod in nullius arbitrium reduci potest (Brev., X, 2).

La vida se divide en tres períodos: el que fue, el que es, el que será. De ellos, el que estamos viviendo es breve, el que vamos a vivir, dudoso, el que hemos vivido, cierto. Pues este último es uno sobre el que la fortuna perdió sus derechos, y que no puede estar sometido al arbitrio de nadie.

Esta afirmación, sin embargo, puede conciliarse con la teoría general del tiempo de los estoicos. El tempus quod est/ quod agimus es el punto de conjunción entre el pasado y el futuro, un punto en constante movimiento hacia adelante; el tempus quod futurum est/ quod acturi sumus es incierto ya que aún no estamos involucrados en él, pero está bajo el control del sapiens porque él elegirá vivir ese periodo de manera racional $\mathrm{y}$, por definición, éticamente, ya que en términos estoicos, lo racional equivale al bien; el tempus quod fuit/ quod egimus es fijo, no sólo porque las acciones humanas no pueden deshacerse, sino también porque el ciclo cósmico no puede ser vuelto hacia atrás. ${ }^{10}$ De este modo, la relación entre el individuo y el tiempo está inextricablemente ligada a su conducta en la vida: si vive racionalmente, de acuerdo con los preceptos estoicos, la vida no le parecerá injustamente corta, sin importar cuán grande o pequeño sea el segmento de tiempo cíclico que su vida ocupe.

La representación del tiempo en toda la tragedia senequeana se caracteriza por la extensión del tempus quod est/ quod agimus más allá de sus límites naturales: en lugar de funcionar como el punto de conjunción momentáneo entre el pasado y el presente, se convierte en un acontecimiento de duración extendida. En parte, esto es producto de la propia forma dramática, en la que los acontecimientos representados coinciden 
con la observación de la audiencia. Pero eso solo no basta para explicar, por ejemplo, la persistencia del adverbio iam en el monólogo con el que Edipo abre la obra del mismo nombre. ${ }^{11}$ En efecto, iam es la palabra que abre la obra: iam nocte Titan dubius expulsa redit / et nube maestum squalida exoritur iubar (Oed., 1-2), "Ya, desterrada la noche, vuelve Titán vacilante y su resplandor se eleva sombrío desde negruzcas nubes." Más adelante, utiliza la forma repetida ${ }^{12}$ del adverbio: iam iam aliquid in nos fata moliri parant (Oed., 28), "ahora mismo los hados se disponen a tramar algo contra mí" (trad. Luque Moreno, Gredos.). El turbio amanecer y lo que Edipo considera que es una maligna maquinación del destino contra él son presentados como acontecimientos que no sólo existen meramente en el tiempo presente, sino que además persisten.

Esta visión del tiempo es compartida por el coro en la primera oda, que describe la naturaleza duradera de la ruina que sufrió Tebas y su casa real a causa de una peste que parece no tener fin: occidis, Cadmi generosa proles, / urbe cum tota (Oed. 110-111), "estás cayendo, noble descendencia de Cadmo, junto con toda la ciudad." Sobre el final del monólogo de Edipo reaparece el adverbio iam intensificado por dudum, que a la vez imparte un sentido de urgencia y cambia el foco hacia el futuro: profuge iamdudum ocius / vel ad parentes (Oed., 80-81), "huye ahora mismo, cuanto antes, aun hacia tus padres." De este modo, Edipo se ha movido desde una extensión del momento presente a una exhortación urgente hacia el futuro. Este deslizamiento temporal es acentuado por la descripción, en otra parte del monólogo, del ocultamiento de los cuerpos celestes: nullum serenis noctibus sidus micat (Oed., 46), "ninguna estrella brilla en el cielo de una noche despejada." Durante el día el sol es brumoso e indistinto; durante la noche las estrellas no son visibles: por tanto, los cuerpos celestes utilizados para medir el tiempo, y para entenderlo racionalmente, se han vuelto poco fiables a causa de la disrupción cósmica. Es por esta razón que Edipo ruega por matura fata, "un hado prematuro", praecurram ut prior / patriam ruentem, neve post omnes cadam / fiamque regni funus extremum mei (Oed., 72-74), "para poder adelantarme a la ruina de mi patria y no caer después de todos y convertirme en la última muerte de mi reino.” Edipo intenta aquí imponer certezas a un futuro que, en el marco temporal del De brevitate vitae, es incierto incluso en el racionalmente ordenado cosmos estoico, aun cuando en un sentido amplio los acontecimientos futuros forman parte de un inexorable ciclo cósmico, predeterminado pero desconocido. ${ }^{13}$

De este modo, lo que Edipo intenta lograr es nada menos que un adynaton cósmico. Incluso en el orden cósmico estoico, en el que el futuro está predeterminado, no está en su potestad imponer un orden cósmico: éste simplemente existiría, y su obligación, bajo los preceptos estoicos, sería alinearse voluntariamente a él. Edipo es igualmente impotente para imponer un orden a un universo desordenado, pero esto es precisamente lo que intenta lograr con la maldición proferida contra el asesino de Layo. Además de invocar a las divinidades tradicionales, invoca a los mismos cuerpos celestes cuya dislocación había señalado en su monólogo inicial:

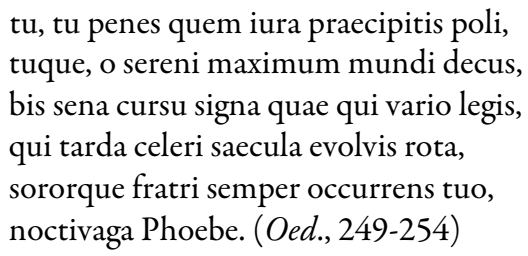

Tú, que tienes las leyes de los cielos vertiginosos; y tú, máxima gloria del cielo despejado, que atraviesas los doce signos en tu cambiante trayectoria y desenrollas las lentas edades con tu veloz rueda; y tú, hermana, que siempre sales al encuentro de tu hermano, noctámbula Febe.

En el cosmos que describe Edipo en su monólogo inicial, no es posible observar el recorrido del sol a través del zodíaco o el recorrido de la luna, ni vemos indicio alguno en los versos siguientes de que se haya despejado el cielo: la sórdida obstrucción de los cielos sigue tan obstinadamente en pie como los estragos de la peste. Edipo, por tanto, invoca - y hace depender de ellas el cumplimiento de su maldición- certezas cósmicas cuya ausencia él mismo ha establecido. 
La propia voz de Edipo, que abría la obra, también la cierra, ${ }^{14}$ y por tanto concluiré mi breve discusión sobre Edipo analizando su monólogo final. El modo imperativo, que implica inherentemente el futuro, domina el pasaje. Se ordena a sí mismo "seguir caminos engañosos" (sequere fallentes vias, Oed., 1047) y "guiar con mano temblorosa tu noche ciega" (caecam tremente dextera noctem rege, Oed., 1049). Al cegarse, Edipo ha internalizado la noche en la cual, al principio de la obra, nada era visible; es esta noche desprovista de cuerpos celestes la que imagina ahora sacar de la ciudad junto con la peste que la estaba afligiendo. Él mismo está seguro de que el orden cósmico se restablecerá luego de su partida: mitior caeli status / post terga sequitur ("una disposición de cielo más benigna/ viene tras de mí" Oed. 1054-1055) - tan seguro está, de hecho, que utiliza el presente, sequitur, en lugar del futuro sequetur, aun cuando la cantidad larga puede ser sustituida al comienzo del segundo pie de un senarius: su uso de un praesens pro futuro sugiere, de este modo, que el buen tiempo y la restauración del orden cósmico que éste representa efectivamente están por llegar. Es su último y desesperado intento de controlar el futuro, lo cual, por supuesto, no puede hacer. Su último imperativo, mecum ite, mecum ("vengan conmigo, vengan," Oed., 1061), está dirigido a los "salvajes Hados" (violenta Fata), "el horrible temblor de la Enfermedad" (horridus Morbi tremor), "Demacración y negra Peste y rabioso Dolor" (Macies et atra Pestis et rabidus Dolor, Oed., 1059-1060). Al colocar a los hados al comienzo de la lista, Edipo revela que su delirio esencial se mantiene intacto: así como cree que puede conocer el futuro, cree que puede controlar el destino, lo que se revela como el más grande adynaton estoico. Tal como lo dice Séneca en Ep., CVII, 11, ducunt volentem fata, nolentem trahunt - "los hados conducen a quien los acepta, y arrastran a quien no los acepta." El sapiens estoico se ocupa de alinearse con el orden cósmico de manera consciente y voluntaria; el estoico fracasado se ve forzado a hacerlo. Edipo imagina ser el primero, pero actúa invariablemente como el último.

Si el monólogo inicial de Edipo nos confronta con un universo ya inestable, el de Medea supone una intacta concepción pre-estoica del universo, pues jura por las divinidades celestiales e infernales tradicionales. Este universo tradicional es no sólo inherentemente pre-estoico, sino anti-estoico, ya que no hay lugar en el estoicismo para el caos o para el inframundo, pero al menos es un universo coherente. Uno esperaría que de esta cosmología emergiera una concepción tradicional del tiempo, y de hecho eso mismo ocurre: cuando Medea lanza sus maldiciones contra Jasón y jura vengarse de él, presupone la capacidad de la agencia humana tanto para afectar los acontecimientos futuros como para invocar la ayuda sobrenatural para llevarlos a cabo. Sin embargo, la propia Medea complica el problema del tiempo ya en la primera línea del monólogo que abre el segundo acto: occidimus: aures pepulit hymenaeus meas (Med., 116). Pepulit es un auténtico perfecto de acción terminada en el presente; "el himno nupcial ha sacudido mis oídos", dice Medea refiriéndose al himno nupcial de Jasón y Creúsa. Pero occidimus es ambiguo: ¿se refiere Medea a que su colapso es completo, ${ }^{15}$ o que está sucediendo, habiendo sido iniciado por el himno nupcial?

La cuestión del tiempo pasa temáticamente a un primer plano en el diálogo con la nutrix. En respuesta a la promesa de venganza de Medea, fugiam et ulciscar prius (Med.172), "Me iré al exilio y antes me vengaré," la nutrix dispensa lo que podría interpretarse como un consejo estoico: compesce verba, parce iam, demens, minis / animosque minue; tempori aptari decet ("refrena las palabras, abstente ya de amenazas, insensata, y abate tu ira: es conveniente adaptarse a las circunstancias," Med., 174-175). Una de las muchas connotaciones posibles de tempus es algo así como "las circunstancias presentes", reflejada en mi elección (siguiendo a John Fitch) del plural; a nivel del sentido, la nutrix está aconsejando a Medea aceptar condiciones que escapan a su control. Pero la nutrix también se refiere a Medea como demens, "loca, irracional", y le aconseja suprimir su ira. Sabemos que, para los estoicos, la ira es la más destructiva de todas las emociones, ya que implica una completa sumisión a lo irracional; de este modo, las palabras de la nutrix pueden ser interpretadas como un intento de persuadir a Medea de volverse un actor racional en el sentido estoico. A partir de esta lectura, aptari tempori sería un reflejo del principio estoico naturam sequi, seguir a la naturaleza; este principio expresa no sólo la comprensión de (sino también la sumisión a) este universo estoico racional, lo que además también implica necesariamente aceptar la realidad del eterno retorno temporal. 
Pero la propia Medea tiene una concepción diferente del universo: en su diálogo con Creonte declara: confide regnis, cum levis magnas opes / buc ferat et illuc casus ("confía en tu reinado, mientras/porque/ aunque el voluble azar lleva grandes riquezas de acá para allá,” Med., 221-222). Es debido a la volubilidad de la suerte - una visión anti-estoica- que Medea considera que el pasado puede regresar. El coro complica aún más la cuestión en la oda subsiguiente, que comienza con el antiguo motivo de la impiedad del primer navegante: ${ }^{16}$ audax nimium qui freta primus / rate tam fragili perfida rupit (Med., 301-302, "demasiado atrevido fue el primero que surcó el mar traicionero con una barca tan frágil," una clara alusión a Horacio, Carm., I, $3^{17}$ ). En la visión del coro, la ignorancia de las estrellas era una característica de la "prelapsaria" 18 edad de oro anterior a la navegación: nondum quisquam sidera norat, / stellisque, quibus pingitur aether, / non erat usus ("aún nadie entendía los astros, ni hacía uso de las estrellas que enriquecen el cielo," Med., 309-311). Los cuerpos celestes existían, pero la humanidad no sabía guiarse en la navegación a través de ellos. En otras palabras, el coro reconoce la existencia de un orden cósmico, pero afirma que la humanidad estaba mejor al no comprenderlo - que la humanidad era más dichosa con su primitiva concepción del tiempo, definida por una comprensión limitada sobre los movimientos regulares de los cuerpos celestes, que no se extendía más allá de las noches, los días y las estaciones. La nave que destruyó la inocencia humana fue la Argos - y esa nave trajo a Medea, "un mal peor que el propio mar y una mercancía digna de la primera nave" (maiusque mari Medea malum, / merces prima digna carina, Med., 363-364, sigue diciendo el coro).

Medea, en el acto siguiente, conecta temporariamente su propia ira con el orden cósmico:

dum terra caelum media libratum feret, nitidusque certas mundus evolvet vices, numerusque harenis derit, et solem dies, noctem sequentur astra, dum siccas polus versabit Arctos, flumina in pontum cadent, numquam meus cessabit in poenas furor, crescetque semper. (Med., 401-407)

Mientras la tierra en el centro sostenga en equilibrio el cielo, mientras el brillante firmamento desenrolle sus certeras vueltas y no haya cálculo posible para la arena, mientras el día acompañe al sol y las estrellas asistan a la noche, mientras el Polo haga girar a las secas Osas y los ríos caigan al mar, nunca cesará mi furia su progreso hacia la venganza; más bien, crecerá cada vez más.

Todos estos verbos están en futuro; de este modo Medea condiciona su furia a la certeza de que la coherencia cósmica seguirá prevaleciendo. ${ }^{19}$

Más tarde, sin embargo, su brujería transforma este orden en confusión:

et evocavi nubibus siccis aquas

egique ad imum maria. et Oceanus graves

interius undas aestibus victis dedit;

pariterque mundus lege confusa aetheris

et solem et astra vidit, et vetitum mare

tetigistis, Ursae. temporum flexi vices. (Med., 754-759)

E hice caer agua de las nubes sin lluvia y conduje el mar a sus profundidades. Y Océano retiró sus potentes olas cuando sus mareas fueron dominadas. Con las leyes del cielo turbadas, el mundo ha visto al mismo tiempo el sol y las estrellas, y ustedes, Osas, han tocado el mar prohibido. He cambiado la sucesión de las estaciones (alternativa: he torcido el orden del tiempo).

Esta imaginería nos es familiar de muchas representaciones antiguas de la brujería; la idea de que una bruja puede alterar el orden cósmico está extendida al menos desde la época helenística en adelante. Aquí, sin embargo, no es mero cliché: la ira de Medea ha evolucionado de estar supeditada al orden cósmico a demoler este orden por medio de las fuerzas sobrenaturales que ella comanda. Junto a este orden, el propio tiempo ha sido torcido. De este modo, cuando Jasón, al contemplar la huida de Medea hacia el cielo en el carro alado tirado por serpientes, proclama la inexistencia de la divinidad en los últimos versos de la obra (per alta vade 
spatia sublime aetheris; / testare nullos esse, qua veheris, deos "ve por los aires a través de las altas extensiones del cielo; da testimonio de que donde eres transportado, no hay dioses” Med. 1026-1027), reconoce la escala cósmica del poder de Medea. La tragedia senequeana a menudo nos enfrenta con una distopía impersonal y específicamente anti-estoica de desorden cósmico; aquí, sin embargo, Medea, cuya ira la ubica en el extremo opuesto del ideal ético estoico, resulta no ser una mera destructora de certezas estoicas, sino la creadora y la soberana del caos universal.

Llegamos finalmente a Thyestes, que comienza con otra erupción del inframundo: la Furia, un agente ctónico de lo irracional, afirma su control sobre el futuro a través del fantasma inicialmente reacio de Tántalo. Cuando el fantasma por fin cede, en el verso 100, lo hace pronunciando un extra metrum $^{20}$ : sequor -en presente, pero con un elemento de praesens pro futuro. La primera oda coral narra los crímenes de Tántalo, que pertenecen al pasado inalterable. El voto de venganza de Atreo, como el de $\mathrm{Medea}^{21}$ - de hecho, al igual que la mayoría de los votos de venganza- implica nuevamente un intento de afirmar el control del futuro: age, anime, fac quod nulla posteritas probet, / sed nulla taceat. Aliquod audendum est nefas / atrox, cruentum ("Vamos, espíritu mío, haz lo que ninguna edad futura aprobará y lo que ninguna edad futura pueda callar. Hay que atreverse a algún crimen indecible, salvaje y sangriento," Thy., 192-194). Atreo pronto sigue esta declaración, sin embargo, con un reconocimiento de la pérdida del control actual: fateor. tumultus pectora attonitus quatit/penitusque volvit; rapior et quo nescio, / sed rapior ("Lo reconozco, una turbación enloquecida sacude mi pecho y lo revuelve por dentro; soy arrastrado, no sé adónde, pero soy arrastrado,” Thy., 260-262). Todos los verbos están aquí en auténtico tiempo presente: Atreo describe lo que está sucediéndole en el presente, y el cumplimiento de sus maquinaciones para el futuro depende precisamente de su falta de control sobre estos acontecimientos presentes.

Hasta ahora, la obra nos ha ofrecido el universo habitual de la tragedia senequeana, en el cual lo irracional domina todas las cosas, incluido el tiempo. Por tanto, un gran golpe de ironía dramática hace su aparición cuando el coro, al final del acto 3, logra convencerse, si bien temporariamente, de que el orden cósmico ha sido reestablecido:

iam minae saevi cecidere ferri,

iam silet murmur grave classicorum,

iam tacet stridor litui strepentis:

alta pax urbi revocata laetae est. (Thy., 573-576)

Ahora la amenaza del cruel hierro ha disminuido; ahora hace silencio el poderoso estruendo de las trompetas; también ahora calla el chillido del clarín retumbante: se ha hecho regresar una paz profunda a la ciudad alegre.

El coro se traslada desde una acción terminada en el presente (cecidere) al presente inmediato (tacet, silet) para volver a las acción terminada (revocata est), combinando de este modo las nociones de compleción e inmutabilidad con una inmediatez enfatizada por la insistente anáfora del iam. ${ }^{22}$ Pero más tarde, en la misma oda, el coro se desdice ${ }^{23}$ y reconoce la no permanencia de las cosas: nulla sors longa est: dolor ac voluptas / invicem cedunt: brevior voluptas. / ima permutat levis hora summis ("Ningún estado de cosas es duradero: el dolor y el placer ceden uno al otro; el placer es más breve. Una hora fugaz hace que lo alto y lo bajo intercambien lugares," Thy., 596-598). El final de la oda da a este pesimismo una de las más altas expresiones poéticas de toda la tragedia senequeana:

nemo confidat nimium secundis, nemo desperet meliora lassis: miscet haec illis prohibetque Clotho stare Fortunam, rotat omne fatum. nemo tam divos habuit faventes, crastinum ut posset sibi polliceri. res deus nostras celeri citatas turbine versat. (Thy., 615-622) 
Que nadie confíe demasiado en circunstancias favorables; cuando las circunstancias sean desfavorables, que nadie pierda las esperanzas de cosas mejores: Cloto mezcla ambas cosas, prohíbe a la Fortuna permanecer en un lugar y hace girar cada destino. Nadie ha tenido dioses tan favorables a él como para poder asegurarse el mañana. Dios apresura nuestros asuntos y los hace girar en un torbellino.

El verso nemo desperet meliora lassis, que asegura, en tiempo adversos, la inexorabilidad de la llegada de tiempos mejores, es la única nota contraria en un pasaje que de otro modo sería abrumadoramente pesimista. El coro, como buenos estoicos, ve el tiempo como un ciclo, pero el ciclo es intrínsecamente maligno y, como sugiere la metáfora del torbellino, en última instancia caótico.

El coro llega a una amarga conclusión al final del cuarto acto, durante el cual el nuntius ha utilizado mayormente el presente histórico para narrar el sacrificio de los hijos de Tiestes llevado a cabo por Atreo, trayendo por tanto estos actos terminados a un presente continuo: Solitae mundi periere vices, / nibil occasus, nibil ortus erit (Thy., 813-814), se lamenta el coro; "los ciclos habituales del mundo han muerto; no habrá ocaso ni nacimiento del sol." Ahora ni siquiera se cumple el ciclo maligno de la oda anterior; incluso el ciclo astronómico más rudimentario, el curso diario del sol, será abolido y, por tanto, volverá imposibles aun los modos más primitivos de medir el tiempo. Un poco más adelante en la oda (Thy., 835 ss.), el coro contempla las estrellas cayendo del cielo en una especia de ekpyrōsis inversa. Finalmente, en el verso 878, se pregunta: in nos aetas ultima venit? (“'ha llegada a nosotros la edad final?”). Por decirlo de otro modo: ¿es este el fin del tiempo mismo?

En la medida en que la representación del tiempo en la tragedia de Séneca es coherente, podemos decir que su representación del tiempo es coherentemente inestable; esto está sin duda en consonancia con el nihilismo apocalíptico del cosmos senequeano. En cierto nivel, los personajes trágicos senequeanos son paradigmas negativos de la relación humana con el tiempo que actúan precisamente del modo que el De brevitate vitae prohíbe. Sin embargo, en un sentido más amplio, obedecen al principal mandato estoico de seguir la naturaleza. Ese mandato procede del supuesto de que la naturaleza es racional; en un esquema ético en el que lo racional es el bien, es siguiendo a la naturaleza que uno lucha por el ideal ético. Los personajes trágicos de Séneca siguen a la naturaleza, pero en un universo irracional. De la adecuación a un cosmos enloquecido, solo puede resultar la locura; y dado que el tiempo, en tal cosmos, está distorsionado y es grotesco, los habitantes de este universo que se ajustan a su naturaleza logran una percepción del tiempo que es confusa, deformada, perversa $-\mathrm{y}$ trágicamente precisa. ${ }^{24}$

\section{Traducción:}

\section{Martín Vizzotti}

Universidad Nacional de La Plata

vizzottim@gmail.com

\section{Pablo Martínez Astorino}

Conicet/ Universidad Nacional de La Plata

pmastorino@gmail.com

\section{Notas}

1 De Romilly, J., Time in Greek Tragedy, Ithaca, NY, Cornell University P., 1968, 4.

2 De Romilly, Time in Greek Tragedy, 59-61.

3 De Romilly, Time in Greek Tragedy, 88.

4 De Romilly, Time in Greek Tragedy, 141.

5 Para un ejemplo reciente, véase Kohn, T., "Who Wrote Seneca’s Plays?”, CW 93. 3, 2003, 271-280.

6 Fitch. J. G., (ed./ trad.), Seneca, Tragedies II: Oedipus, Agamemnon, Thyestes, Hercules on Oeta, Octavia. Loeb Classical Library 78, Cambridge, MA- London, Harvard University P., 2004, 333. 
7 Fitch, J. G., "Sense-Pauses and Relative Dating in Seneca, Sophocles, and Shakespeare", AJPh 102, 1981, 289-307, seguido por Marshall, C.W., "The Works of Seneca the Younger and Their Dates”, en: Damschen, G. -Heil, A., Brill's Companion to Seneca: Philosopher and Dramatist, Leiden, Brill, 2013, 37-41.

8 Williams, G. D. (ed.), Seneca: De otio, De brevitate vitae, Cambridge, Cambridge University P., 2003, 20.

9 Arnim, H. F. von., Stoicorum Veterum Fragmenta (SVF). 4 vols., Leipzig, De Gruyter, 1903-24.

10 Cf. la observación de Edwards ("Ethics V: Death and Time”, en: Damschen-Heil, Brill's Companion to Seneca, 325) de que Séneca en Brev., sigue un "modelo cósmico del tiempo."

11 Como advierte Boyle, A. J. (ed./ trad., Seneca: Oedipus, Oxford, Oxford University P., 105 ad loc.), iam es una de las palabras más frecuentes en la obra.

12 "Duplicación emotiva," como observa Boyle (Seneca: Oedipus, 119 ad loc.) este tipo de geminación es "bei Seneca beliebt" (Töchterle, K. ed./ trad., Lucius Annaeus Seneca: Oedipus, Heidelberg, 1994, 160 ad loc.).

13 Sin embargo, no incognoscible: de ahí la aceptación estoica de la adivinación y los augurios.

14 Sklenár. R., Plant of a Strange Vine: > Oratio Corrupta< and the Poetics of Senecan Tragedy. Beiträge zur Altertumskunde 363, Berlin-Boston, De Gruyter, 2017, 53.

15 Tal como interpreta Boyle, Seneca: Oedipus, 155 ad loc.: "estamos muertos."

16 Wessels, A., Ästhetisierung und ästhetische Erfahrung von Gewalt: Eine Untersuchung zu Senecas Tragödien. Bibliothek der klassischen Altertumswissenschaften (N.F.) Reihe 2, Band 137, Heidelberg, Universitätsverlag Winter, 2014, 75-76.

17 Costa, C. D. N. (ed.), Seneca: Medea, Oxford, Oxford University P., 1973, 99 ad loc.; Boyle, Seneca: Oedipus, 209 ad loc.

18 Boyle, Seneca: Oedipus, 211 ad 309.

19 Costa, Seneca: Medea, 109 ad loc.

20 Thy., 100 es uno de los cuatro ejemplos de este fenómeno en todo el corpus de Séneca tragicus; los otros son Phaed., 605, Pho., 319, and Tro., 1103 (Boyle, A. J., ed./ trad., Seneca: Thyestes, Oxford, Oxford University P., 2017, 141 ad loc.).

21 Tarrant, R. (ed.), Seneca's Thyestes. American Philological Association Textbook Series 11, Atlanta, Scholars P., 1985, 119 ad loc.; Boyle, Seneca: Thyestes, 177-178 ad loc.

22 Boyle, Seneca: Thyestes, 300 ad loc.

23 Tarrant, Seneca's Thyestes, 176 ad 596-606; Boyle, Seneca: Thyestes, 310 ad 615-622.

24 Tuve el honor de presentar una primera versión de este ensayo en la Conferencia Inaugural de las IX Jornadas de Estudios Clásicos y Medievales en la Universidad Nacional de la Plata en agosto de 2019. Quisiera expresar mi profunda gratitud a mis anfitriones en esa ocasión, quienes me brindaron la más grata hospitalidad que jamás haya experimentado — verdadera 乡̇vi $\alpha$ en su más alta tradición. 